\title{
An Improved Technique for Gray Scale Image Enhancement based on Multiscale Morphological Approach
}

\author{
Pawandeep Kaur \\ Computer Science Department B.M.S.C.E, Muktsar \\ India (Punjab)
}

\author{
Mandeep Singh \\ Computer Science Department B.M.S.C.E, Muktsar \\ India (Punjab)
}

\begin{abstract}
A method for enhancing the contrast of gray scale images using multiscale mathematical morphology is presented in this paper. The conventional theoretical concept of enhancing image has been extended from mathematical morphology to multiscale mathematical morphological approach. Gray scale images sometimes suffer from lack of contrast. So to enhance image efficiently a multiscale top hat transform based algorithm is proposed. In this algorithm multiscale structuring element of same shape and increasing size is used. By using multiscale top hat transform both white and black features at various scales of gray scale image are extracted and these features are added together to find the final enhanced image. For implementing scheme different morphological towers are built. Experiment has been performed on a set of raw gray scale images for testing efficiency both qualitatively and quantitatively and result is also compared with other state of art techniques and gets better result.
\end{abstract}

\section{General Terms}

Multi Scaling, Mathematical Morphology

\section{Keywords}

Gray-scale image, Image enhancement, Multiscale

morphology, Tophat transform

\section{INTRODUCTION}

Image enhancement is used to improve the interpretability for human viewers. Image enhancement technique can be categorized in two ways including spatial domain technique in which the pixels are directly operated and frequency domain technique in which operations are performed on the Fourier transform of the query image[2].

For noise removal many algorithms has been proposed like histogram based algorithms, fuzzy logic based, diffusion based algorithms etc. To achieve a good result for image enhancement multiscale top hat transform is applied on gray scale query image [4].In morphology image is treated according to set theoretic concept of shape. Objects in an image are considered as set and operations are performed between two sets: the object and the structuring element (SE). According to purpose of associated application shape and size of SE is defined. In morphology erosion and dilation are basic operations and opening (closing) is sequential combination of erosion (dilation)[1].

\section{MATHEMATICAL MORPHOLOGY}

In the field of image processing, computer vision and analysis, mathematical morphology is a powerful tool. In morphology objects presented in the image are treated as sets of points and operations are conducted between two sets: the object and SE. two basic operations of mathematical morphology are erosion and dilation[1]. Let $a$ and $\mathrm{s}$ represent a gray- level image and structuring element respectively. The dilation erosion and of $a(\mathrm{x}, \mathrm{y})$ by $\mathrm{s}(\mathrm{u}, \mathrm{v})$ are denoted as follows, respectively[3]

$$
\begin{aligned}
& a \oplus \mathrm{s}=\max _{i, j}(a(x-u, y-v)+\mathrm{s}(u, v)) \\
& a \Theta \mathrm{s}=\min _{i, j}(a(x+u, y+v)-\mathrm{s}(u, v))
\end{aligned}
$$

Opening and closing [3] are defined as, respectively

$a \mathrm{O} \mathrm{s}=(a \Theta \mathrm{s}) \oplus \mathrm{s}$
$a \bullet s=(a \oplus s) \Theta s$

Tophat transform containing white Tophat and black Tophat denoted by WTH and BTH are respectively defined as follows:

$$
\begin{aligned}
& \operatorname{WTH}(\mathrm{x}, \mathrm{y})=a(\mathrm{x}, \mathrm{y})-a \text { o s }(\mathrm{x}, \mathrm{y}) \\
& \operatorname{BTH}(\mathrm{x}, \mathrm{y})=a \bullet \mathrm{s}(\mathrm{x}, \mathrm{y})-a(\mathrm{x}, \mathrm{y})
\end{aligned}
$$

Corresponding to the size of SE white regions of image are usually smoothen by opening. Hence WTH is used to extract white regions in the image. Similarly using the closing operation black regions of image are smoothen corresponding to the size of use SE. So black regions of image are usually extracted by BTH[4].

\section{MULTISCALE MATHEMATICAL MORPHOLOGY}

White and black image features are contained in different scales of image. If these white and black features at all the scales could be extracted using scalable structuring element(SE) then query image could be enhanced more effectively. Such a method where varying scale structuring element is used is known as multiscale morphology[4,3]. Multiscale opening and closing operations are defined respectively, as 
$a \mathrm{o} \mathrm{s}=(a \Theta \mathrm{ns}) \oplus \mathrm{ns}$

$a \bullet s=(a \oplus n s) \Theta \mathrm{ns}$

Where $\mathrm{n}$ is integer, showing the scale factor of SE[1].

Scale- specific white and black features are extracted using structuring element at different scale. Multiscale WTH and BTH are described as follows

$\mathrm{WTH}(\mathrm{x}, \mathrm{y})=a(\mathrm{x}, \mathrm{y})-(a \mathrm{o} \mathrm{ns})$

$\operatorname{BTH}(\mathrm{x}, \mathrm{y})=(a \bullet \mathrm{ns})-a(\mathrm{x}, \mathrm{y})$

Where $\mathrm{s}$ is structuring element of definite shape and $\mathrm{n}$ is an integer representing the scale factor of structuring element.

\section{METHODOLOGY}

Difference between original image and its mean image is amplified using local statistics in a contrast stretching method as follows

$\tilde{a}(x, y)=\bar{a}(x, y)+m[a(x, y)-\bar{a}(x, y)]$

$\mathrm{m}$ is global amplification factor having value greater than1.WTH decomposes image into two parts as follows

$a(\mathrm{x}, \mathrm{y})=(a \circ \mathrm{o} \mathrm{s})(\mathrm{x}, \mathrm{y})+[a(\mathrm{x}, \mathrm{y})-(a$ o ns $)(\mathrm{x}, \mathrm{y})]$
part 1

All the features of $a(\mathrm{x}, \mathrm{y})$ which are smaller than $\mathrm{s}$ are present in part 2. So to measure local contrast in original image we have

$a(x, y)=(a$ os $)(x, y)+m[a(x, y)-(a$ os $)(x, y)]$

now purpose $\mathrm{m}=2$

$a(x, y)=a(x, y)+[a(x, y)-(a$ os $)(x, y)]$

Let $a(x, y)-(a$ os $)(x, y)=A^{o}(x, y)$ i.e features of size less than that of s obtained by bright tophat, as

$\tilde{a}(x, y)=a(x, y) A_{B}^{o}(x, y)$

We use disk structuring element $\mathrm{s}$, then ks is dilated by $\mathrm{s}(\mathrm{k}-1)$ times. Then we can modify expression of features as

$A^{O}\left(\mathrm{x}_{k s} \mathrm{y}\right)=[\mathrm{a}$ o $(\mathrm{k}-1) \mathrm{s}-(\mathrm{a} o \mathrm{ks})]$

$A^{O}(x, y)$ contains bright features of image $a(x, y)$ which are smaller than scale ks but bigger than $(\mathrm{k}-1) \mathrm{s}$. hence modified image can be obtained as

$m$

$a(x, y)=a(x, y)+\sum A^{o}(x, y)$

In same way multiscale dark features (black tophat transform) can be obtained, we can have another modified image.

$m$

$$
\begin{aligned}
& \left.a(x, y)=a(x, y)-\sum_{k=1} A^{c} \underset{k s}{x}, y\right) \\
& A_{k s}^{c}(\mathrm{x}, \mathrm{y})=(\mathrm{s} \bullet \mathrm{ks})-[\mathrm{s} \bullet(\mathrm{k}-1) \mathrm{s}]
\end{aligned}
$$

For enhanced image having both black and white contrast we add (18) \& (19) as

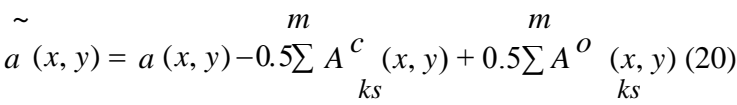

To provide equal weight ages to both bright and dark features contrast multiplier 0.5 is used[2,3].

\section{IMPLEMENTATION}

To implement eq. (20), morphological towers are built[2,3].the image to be enhanced is made to undergo a sequence of gray scale morphological opening operations with a disc structuring element and its higher-order homothetic. Stack which contains he resulting sequence of images is known as opening tower, as identical stack known as closing tower is constructed with multiscale morphological closing of the query image. Therefore $\mathrm{k}^{\text {th }}$ entry in the opening and closing tower represents image opened or closed with structuring element of ks.
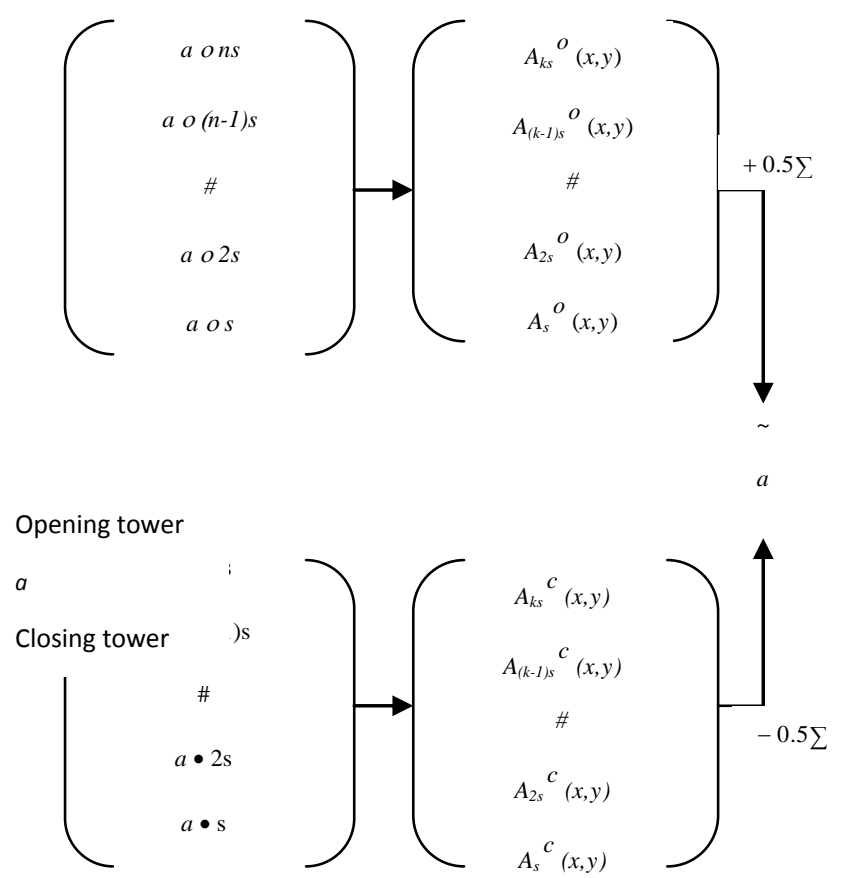

Fig.1 Different Morphological towers 


\section{EXPERIMENTAL RESULTS}

The purposed method has been tested on a set of gray scale images and results have been compared with that of other methods. We have used disk structuring element having smallest size $3 * 3$ and largest size $13 * 13$.

From these images we can see differences of these methods clearly. Image obtained better contrast when black and white features are enhanced using improved multiscale morphological method mentioned above. By using other methods for example, histogram equalization, Unsharp masking, Image Averaging, Single Scale Filtering the processed images are too bright or too dark or so much smooth and some where more sharp at the edges only, which are not satisfied, so proposed method is used to get better results.

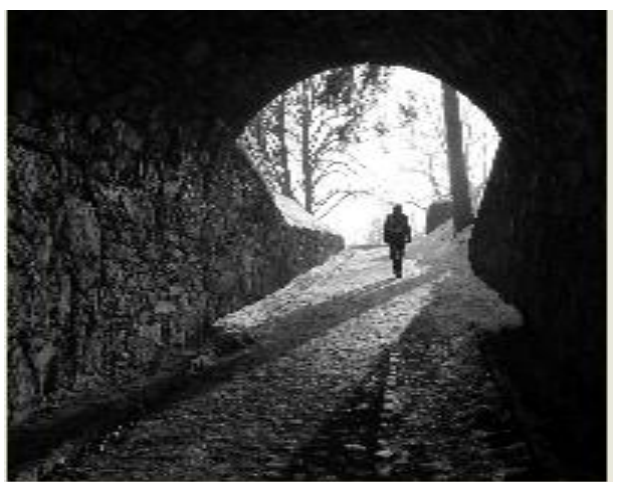

Fig 2 original image

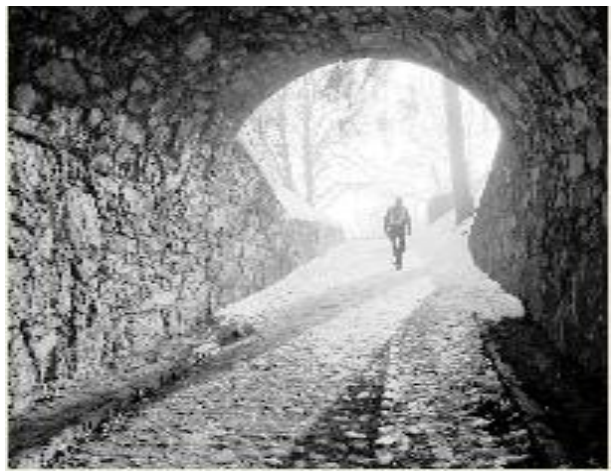

Fig 3 Histogram Equalized Image

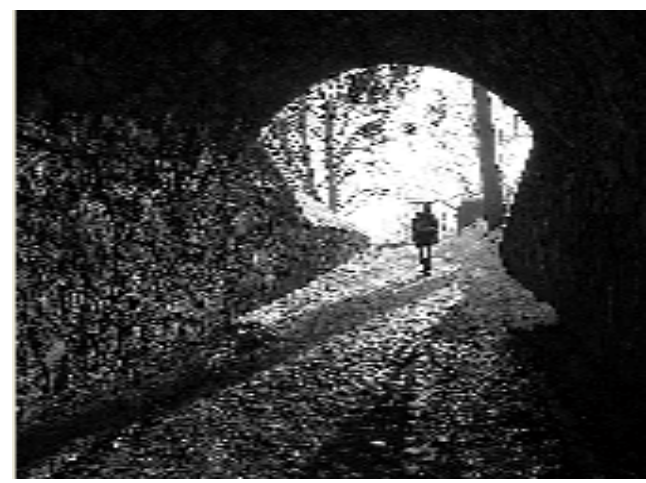

Fig 4 Unsharp Masking

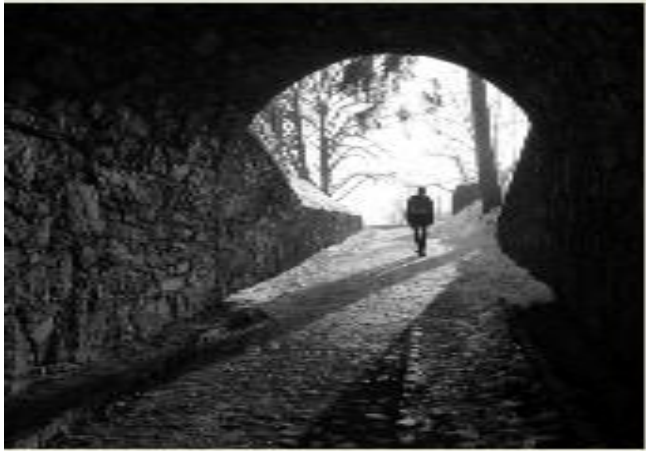

Fig 5 Image Averaging

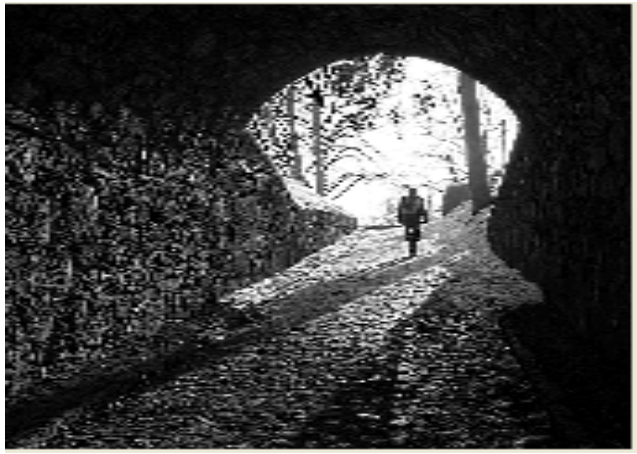

Fig 6 Single Scale Filtering

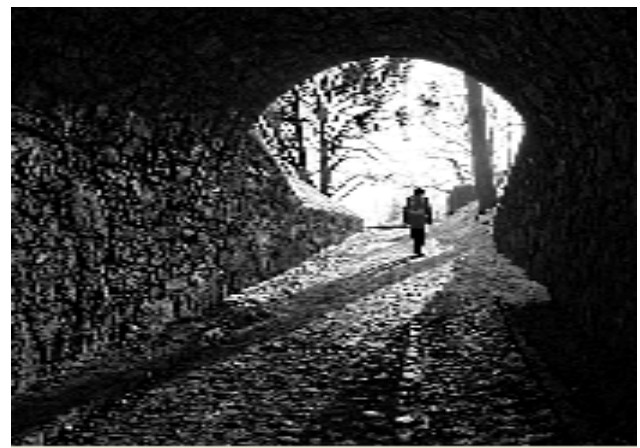

Fig. 7 image after applying proposed method The performance of the enhancement technique is compared through the evaluation of quantitative measures such as DV and BV. An efficient enhancement technique should yield for processed images. Experimental results shown in Table1 validates that results obtained using proposed algorithm are best as compared to those obtained with other state-of-art technique for gray scale images.

Table1. Comparison of DV and BV for input image, stateof-art technique and proposed method of image enhancement

\begin{tabular}{|c|l|c|c|}
\hline $\begin{array}{c}\text { Image } \\
\text { Name }\end{array}$ & \multicolumn{1}{|c|}{$\begin{array}{c}\text { State-of-art } \\
\text { Technique }\end{array}$} & $\begin{array}{c}\text { Detailed } \\
\text { Variance } \\
(\mathrm{DV})\end{array}$ & $\begin{array}{c}\text { Background } \\
\text { Variance } \\
(\mathrm{BV})\end{array}$ \\
\hline \multirow{5}{*}{ Hand.jpg } & Original Image & 71.6371 & 2.93327 \\
\cline { 2 - 4 } & $\begin{array}{l}\text { Histogram } \\
\text { Equalization }\end{array}$ & 66.2397 & 6.72638 \\
\cline { 2 - 4 } & Unsharp Masking & 70.5808 & 7.40856 \\
\hline
\end{tabular}




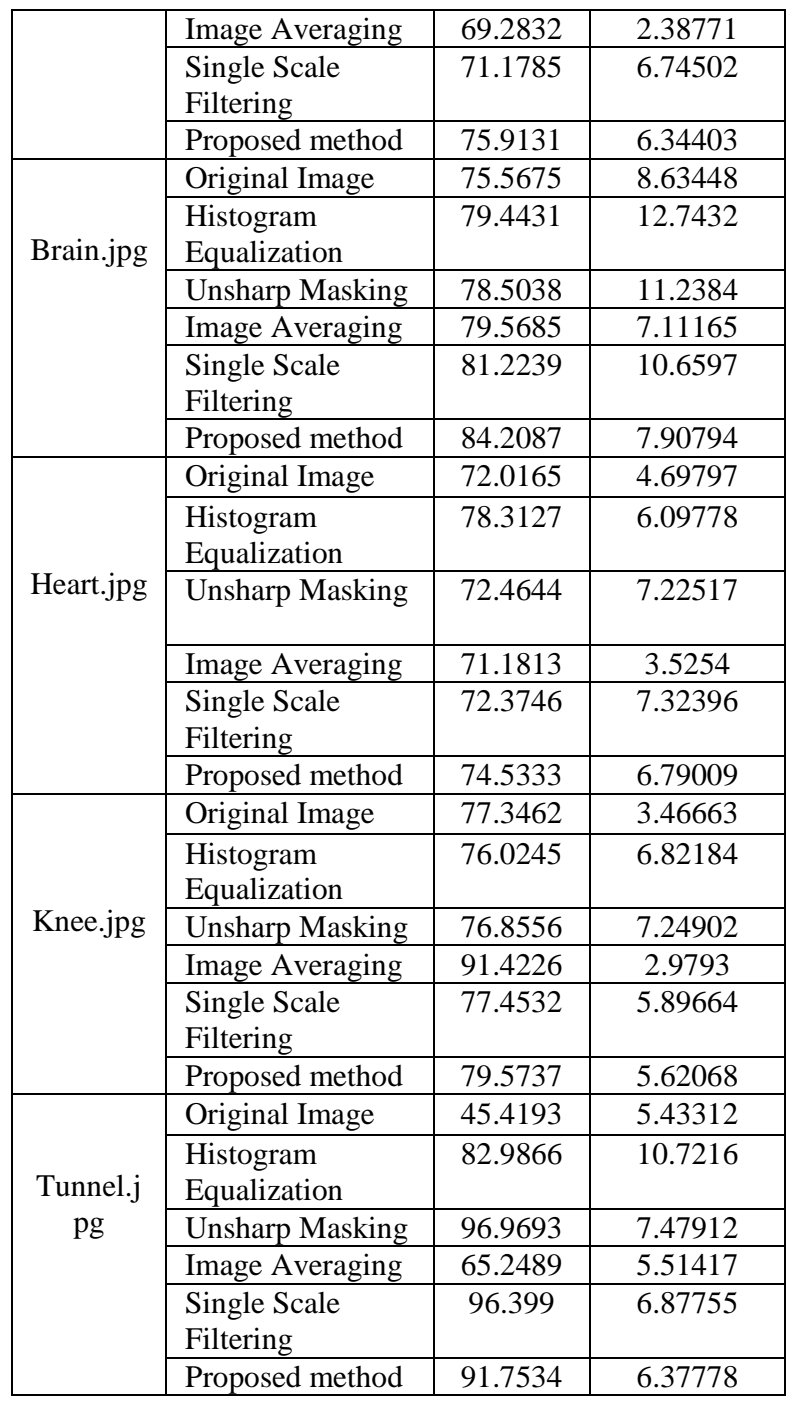

\section{CONCLUSION}

A method for gray scale image enhancement using multiscale mathematical morphology is introduced in this paper. This method is manipulating the intensity of scale specific features in the image by using different scale structuring element, different morphological towers are constructed. By implementing opening and closing towers mutiscale white and black features are extracted, by combining all these features and original image we got final image whose local contrast is enhanced. The purposed method has been implemented on gray scale images and got effective results. It is shown that our findings may be useful to enhance gray scale image.

\section{ACKNOWLEDGMENTS}

The authors greatfully wish to thank Dr. Vikas Chawla, Principal, Dr. Gurbhinder Singh Brar, Dean Academics, Bhai Maha Singh College of Engineering, Sri Muktsar Sahib for their support and constant guidance.

\section{REFERENCES}

[1] Susanta Mukhopadhyay, Bhabatosh Chanda 2000. A multiscale morphological approach to local contrast enhancement. Signal Processing

[2] Computer Vision CITS4240, School of Computer Science \& Software Engineering The University of Western Australia

[3] Zheng Wei, Yang Hua, Sun Hui-sheng, Fan Hong- qi 2007. X-ray Image Enhancement Based on Multiscale morphology. IEEE, Science and Technology Research and Development Program of Hebei Province

[4] Xiangzhi Bai, Fugen Zhou 2000. Multi Scale Top-hat Transform Based Algorithm for Image Enhancement, IEEE Image processing center Beijing University of Aeronautics and Astronautics, Chinna

[5] Yan Wenzhong 2009. Mathematical Morphology based Enhancement for Chromosome Images. IEEE 